\title{
Emittance preservation through density ramp matching sections in a plasma wakefield accelerator
}

\author{
Yujian Zhao $\odot,{ }^{1}$ Weiming An $\odot,{ }^{2,1, *}$ Xinlu Xu ${ }^{1,3, \dagger}$ Fei Li®, ${ }^{1}$ Lance Hildebrand $\odot,{ }^{1}$ \\ Mark J. Hogan, ${ }^{3}$ Vitaly Yakimenko, ${ }^{3}$ Chan Joshi, ${ }^{4}$ and Warren B. Mori ${ }^{1,4}$ \\ ${ }^{1}$ Department of Physics and Astronomy, University of California Los Angeles, \\ Los Angeles, California 90095, USA \\ ${ }^{2}$ Department of Astronomy, Beijing Normal University, Beijing 100875, China \\ ${ }^{3}$ SLAC National Accelerator Laboratory, Menlo Park, California 94025, USA \\ ${ }^{4}$ Department of Electrical Engineering, University of California Los Angeles, \\ Los Angeles, California 90095, USA
}

(Received 26 July 2019; published 3 January 2020)

\begin{abstract}
In plasma wakefield acceleration, the witness beam's emittance needs to be preserved when it propagates through a plasma stage. The plasma includes density ramps at both the entrance and the exit. Using the Wentzel-Kramers-Brillouin solution of a single particle's motion, analytical expressions for the evolution of the beam emittance and the Twiss parameters in an arbitrary adiabatic plasma profile are provided neglecting the acceleration of the beam inside the plasma. It is shown that the beam emittance can be preserved under the matching condition even when the beam has an initial energy spread. It is also shown that the emittance growth for an unmatched beam is minimized when it is focused to the same vacuum plane for a matched beam. The emittance evolution from 3D QuickPIC simulation results agree well with the theoretical results. In the some of the proposed experiments on nearly completed FACET II facility, the matching condition may not be perfectly satisfied and the wake may not be perfectly symmetric. It is shown that for a given set of beam parameters that are consistent with FACET II capabilities, even when the assumptions of the theory are not satisfied, the emittance growth can still be minimized by choosing the optimal focal plane. Last, another issue that may cause emittance growth in realistic plasmas is also examined. When using a lithium plasma source in FACET II experiments a helium buffer gas is used. The plasma is formed from field ionization which can lead to a nonlinear focusing force when there are nonuniform helium ions due to its high ionization potential. For an initial beam emittance of $20 \mu \mathrm{m}$, the helium ionization is found to be small and the witness beam's emittance can be preserved.
\end{abstract}

DOI: $10.1103 /$ PhysRevAccelBeams.23.011302

\section{INTRODUCTION}

During the past two decades of research, a number of impressive advances have been made in the beam-driven plasma wakefield acceleration (PWFA) concept. For instance, experiments have shown that these wakes can sustain accelerating gradients exceeding $50 \mathrm{GeV} / \mathrm{m}$ over $\sim$ meter in length [1], and the acceleration of the witness beam in PWFA can be highly efficient while maintaining a high acceleration gradient and small energy spread [2]. In PWFA, an ultrarelativistic electron beam (the drive beam) is used to form a plasma wake that accelerates a second

\footnotetext{
*anweiming@bnu.edu.cn

†xuxinlu04@gmail.com
}

Published by the American Physical Society under the terms of the Creative Commons Attribution 4.0 International license. Further distribution of this work must maintain attribution to the author(s) and the published article's title, journal citation, and DOI. electron beam (the witness beam) that is properly loaded inside the wake. In the so-called blowout regime, the drive beam density is much higher than the plasma density. The electric field of the drive beam will expel all the plasma electrons away and leave an ion channel (i.e., a bubble) after it. As shown in Fig. 1, when the witness beam is located at a proper position inside the wake, the accelerating field can be flattened in order to preserve the energy spread. At the back of the bubble, where the witness beam is located, not only is there a longitudinal electric field that provides a high acceleration gradient, but there is also a transverse focusing force. In addition, when there is azimuthal symmetry, in these nonlinear wakes the longitudinal electric field (the accelerating field) does not depend on $r$ and the transverse focusing force is linear (proportional to $r$ ), points radially inward, and does not depend on $\xi=c t-z$ inside the bubble [3]. The fact that the accelerating field does not depend on $r$ ensures that the beam particles will not gain additional slice energy spread when undergoing acceleration and betatron oscillations 


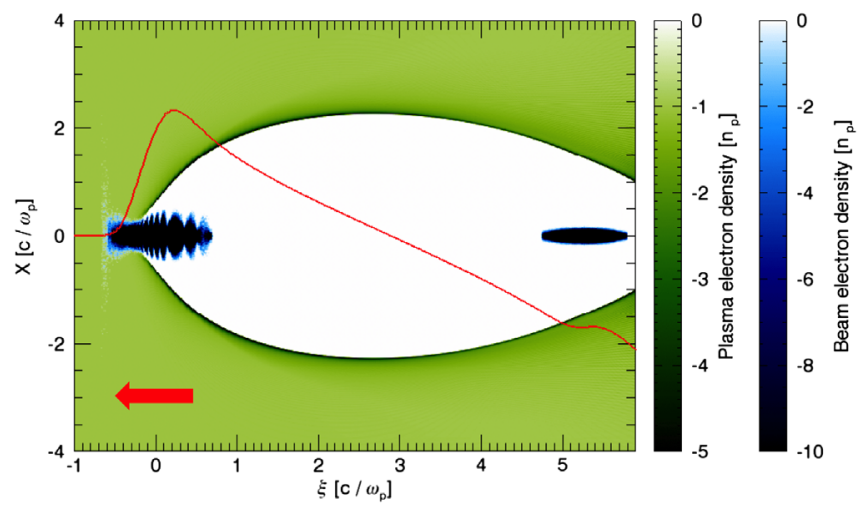

FIG. 1. A snapshot of the drive and witness beam of a sample simulation of a two bunch PWFA. A data is in the $x-\xi$ plane at $y=0$. Both beams (blue) are propagating to the left. The green area shows the unperturbed plasma electron density, the white area is the uniform plasma ions (ion channel/bubble). The red curve is the lineout of the accelerating field $E_{z}$ on the axis (in arbitrary units).

inside the bubble. Furthermore, the fact that the transverse linear focusing force does not depend on $\xi$ ensures that the beam particles at different longitudinal positions will oscillate at the same betatron frequency, if they have the same energy. If one of these properties is satisfied then the Panofsky Wenzel theorem $[4,5]$ guarantees that the other is as well.

When the beam has no energy spread, its emittance is conserved under a linear focusing force inside the symmetric bubble. When the beam has an energy spread, and/or there is acceleration with imperfect beam loading (particles at different longitudinal positions in the beam feel a different accelerating field, $E_{z}$ ), the beam's emittance may increase during its propagation in the plasma. The topic of emittance growth and preservation is very important and is being actively studied [6-13].

Recently, expressions for emittance evolution in uniform plasma, both for cases when the beam does [10] or does not have [11] longitudinal acceleration have been published. It has also been shown that several plasma density profiles provide exact solutions to single particle motion [10,12], therefore the evolution of the beam's Twiss parameters can be calculated and used to match the beam into a plasma. In this paper, we investigate how the emittance grows when a beam is not matched in an adiabatic plasma ramp. In complimentary work, R. Ariniello et al. [13] have recently shown that if a beam is matched to an adiabatic plasma profile, the emittance will oscillate around its initial value with a small amplitude $\left(10^{-4}\right.$ times the initial emittance) for a $2 \%$ energy spread (and the amplitude of oscillations scales as $\sigma_{\gamma}^{2}$ ).

In typical experiments (e.g., the FACET II experiments at SLAC [14]), the plasma density profile is usually fixed with density ramps at the entrance and the exit. Therefore the beam parameters need to be optimized to match the beam to the plasma. It has been shown that if the witness beam is initially matched to the plasma, its emittance can be preserved very well [14]. However, if the witness beam parameters are fixed, it usually cannot be perfectly matched to an arbitrary plasma density ramp. In this paper, we investigate the witness beam's emittance evolution in this situation. We first derive an analytical expression for the beam's emittance evolution in an arbitrary adiabatic plasma profile, assuming the beam has no longitudinal acceleration. This analytical expression can be used to predict the emittance growth when the beam has an energy spread and is not initially matched. This analysis is complimentary to that in [13] where it was assumed that the beam was nearly matched and the emittance growth was small. We also discuss how to choose the relative focal plane by either moving the plasma or the focal position of the beam to minimize the emittance growth for an unmatched beam with fixed parameters. It is found that the beam emittance growth can be minimized when choosing the focal plane to be the vacuum focus for a beam that was matched. Another issue that may cause emittance growth in recently proposed energy doubling of the witness beam experiment at FACET II experiments [1] is the ionization of helium buffer gas when using the Lithium plasma source. Additional self-ionization [15] by the beam can modify the focusing fields in the buffer region. In the last section, we show that under that situation the emittance growth is due to the nonlinear focusing force felt by the beam, which is caused by the nonuniform helium ion density in the plasma. The helium ionization can be minimized by using a $20 \mu \mathrm{m}$ initial emittance witness bunch. Therefore such a bunch can be propagated while gaining energy without measurable emittance growth.

\section{THEORETICAL ANALYSIS OF EMITTANCE EVOLUTION IN ARBITRARY ADIABATIC PLASMA DENSITY PROFILE}

In the blowout regime of PWFA with the assumption of azimuthal symmetry (we will henceforth use this assumption), the focusing force felt by an electron in the witness beam is $\mathbf{F}_{\perp}=-m_{e} \omega_{p}^{2} \mathbf{r} / 2$ (where $m_{e}$ is the electron mass, $\omega_{p}=\sqrt{\frac{n_{p} e^{2}}{\epsilon_{0} m_{e}}}$ is the plasma frequency, $n_{p}$ is the plasma density, $\epsilon_{0}$ is the vacuum permittivity, $e$ is the elementary charge), which is proportional to the radial distance $r$ and independent of $\xi=c t-z$. Therefore the motions of the beam particle in $x$ and $y$ directions are decoupled, and we will only study the beam particle motion in the $x$ direction. If we assume a beam particle's energy is a constant, the equation of motion for this particle is

$$
x^{\prime \prime}(z)+k_{\beta}(z)^{2} x(z)=0
$$

where $z$ is the coordinate along the direction of propagation, $k_{\beta}(z)=\frac{\omega_{p}(z)}{\sqrt{2 \gamma c}}, \omega_{p}(z)$ is the plasma frequency at 
position $z, \gamma$ is the relativistic factor of the beam particle, $c$ is the speed of light. In a uniform plasma, $\omega_{p}(z)$ is a constant, so the solution to Eq. (1) is a simple harmonic oscillation. With a given initial phase space distribution for the beam, we can obtain an analytical expression for the emittance evolution $[10,11]$. For nonuniform plasma, there is no general analytical solution to equation (1). However, as long as the plasma density is changing adiabatically, i.e.,

$$
\frac{\left|k_{\beta}^{\prime}(z)\right| \frac{2 \pi}{k_{\beta}(z)}}{k_{\beta}(z)} \ll 1
$$

or

$$
\frac{\pi}{k_{\beta}(z) n_{p}(z)}\left|\frac{d n_{p}(z)}{d z}\right| \ll 1
$$

we can use Wentzel-Kramers-Brillouin (WKB) method [16] to get an approximate solution to Eq. (1), and calculate the emittance evolution with the WKB solution.

The WKB solution to Eq. (1) is

$$
\begin{aligned}
x(z)= & x(0) \frac{\sqrt{\beta_{m}(z)}}{\sqrt{\beta_{m}(0)}} \cos [\phi(z)] \\
& +\sqrt{\beta_{m}(z) \beta_{m}(0)}\left[x^{\prime}(0)+\frac{\alpha_{m}(0)}{\beta_{m}(0)} x(0)\right] \sin [\phi(z)]
\end{aligned}
$$

where

$$
\beta_{m}(z)=1 / k_{\beta}(z), \quad \alpha_{m}(z)=-\frac{1}{2} \frac{d \beta_{m}(z)}{d z}
$$

are the Twiss parameters for a single particle in an adiabatically changing profile, and $\phi(z)=\int_{0}^{z} k_{\beta}(s) d s$ is the phase advance. $x(0)$ and $x^{\prime}(0)$ are the initial values for the beam particle. Then the adiabatic condition (2) can be simplified to [13]

$$
\left|\alpha_{m}(z)\right| \ll 1
$$

We note that if the plasma density profile is $n_{p}(z)=\frac{n_{p 0}}{(1+z / l)^{4}}$ (where $l$ is a constant), then Eq. (1) has an exact solution [12], which is the same as its WKB solution described in equation (3).

For brevity, we henceforth denote $x(z)$ by $x, x(0)$ by $x_{i}$, $\beta_{m}(z)$ by $\beta_{m}, \beta_{m}(0)$ by $\beta_{m i}, \alpha_{m}(z)$ by $\alpha_{m}, \alpha_{m}(0)$ by $\alpha_{m i}, \phi(z)$ by $\phi$. From (3) and its derivative, we can obtain

$$
\left(\begin{array}{c}
x \\
x^{\prime}
\end{array}\right)=M\left(\begin{array}{c}
x_{i} \\
x_{i}^{\prime}
\end{array}\right)=\left(\begin{array}{ll}
M_{11} & M_{12} \\
M_{21} & M_{22}
\end{array}\right)\left(\begin{array}{c}
x_{i} \\
x_{i}^{\prime}
\end{array}\right)
$$

where $M$ is the transport matrix and

$$
\begin{aligned}
& M_{11}=\sqrt{\frac{\beta_{m}}{\beta_{m i}}}\left(\cos \phi+\alpha_{m i} \sin \phi\right) \\
& M_{12}=\sqrt{\beta_{m} \beta_{m i}} \sin \phi \\
& M_{21}=\frac{\left(\alpha_{m i}-\alpha_{m}\right) \cos \phi-\left(1+\alpha_{m i} \alpha_{m}\right) \sin \phi}{\sqrt{\beta_{m} \beta_{m i}}} \\
& M_{22}=\sqrt{\frac{\beta_{m i}}{\beta_{m}}\left(\cos \phi-\alpha_{m} \sin \phi\right)}
\end{aligned}
$$

The geometric emittance is defined as

$$
\epsilon=\sqrt{\left\langle x^{2}\right\rangle\left\langle x^{\prime 2}\right\rangle-\left\langle x x^{\prime}\right\rangle^{2}}
$$

where \langle\rangle is the ensemble average. It then follows that (see Appendix A for details)

$$
\begin{aligned}
\left\langle x^{2}\right\rangle & =\left\langle\left(M_{11} x_{i}+M_{12} x_{i}^{\prime}\right)^{2}\right\rangle \\
& =\epsilon_{i} \beta_{m}\left(A+B_{1} C+B_{2} S\right),
\end{aligned}
$$

where:

$$
\begin{aligned}
A & =\frac{\beta_{i} \gamma_{m i}+\gamma_{i} \beta_{m i}-2 \alpha_{i} \alpha_{m i}}{2}, \\
B_{1} & =\frac{\beta_{i}}{\beta_{m i}}-A=\frac{\beta_{i}}{\beta_{m i}}-\frac{\beta_{i} \gamma_{m i}+\gamma_{i} \beta_{m i}-2 \alpha_{i} \alpha_{m i}}{2}, \\
B_{2} & =\frac{\beta_{i}}{\beta_{m i}} \alpha_{m i}-\alpha_{i}, \\
C & =\int d \phi f_{\phi}(\phi) \cos 2 \phi, \\
S & =\int d \phi f_{\phi}(\phi) \sin 2 \phi,
\end{aligned}
$$

and $\epsilon_{i}=\sqrt{\left\langle x_{i}^{2}\right\rangle\left\langle x_{i}^{\prime 2}\right\rangle-\left\langle x_{i} x_{i}^{\prime}\right\rangle^{2}}, \beta_{i}=\left\langle x_{i}{ }^{2}\right\rangle / \epsilon_{i}, \gamma_{i}=\left\langle x_{i}^{\prime 2}\right\rangle / \epsilon_{i}$, $\alpha_{i}=-\left\langle x_{i} x_{i}^{\prime}\right\rangle / \epsilon_{i}$ are the beam's initial geometric emittance and Twiss parameters, $\gamma_{m}=\left(1+\alpha_{m}^{2}\right) / \beta_{m}$, and $f_{\phi}(\phi)$ is the distribution function for the beam particles' phase advance. For a beam with no energy spread, $f_{\phi}(\phi)=\delta\left(\phi-\phi_{0}\right)$ where $\phi_{0}=\int_{0}^{z} k_{\beta}(s) d s=\int_{0}^{z} \frac{\omega_{p}(s)}{\sqrt{2 \gamma c}} d s$.

We can also obtain

$$
\begin{aligned}
\left\langle x^{\prime 2}\right\rangle= & \epsilon_{i}\left[A \gamma_{m}+\frac{-B_{1}-2 B_{2} \alpha_{m}+B_{1} \alpha_{m}^{2}}{\beta_{m}} C\right. \\
& \left.+\frac{-B_{2}+2 B_{1} \alpha_{m}+B_{2} \alpha_{m}^{2}}{\beta_{m}} S\right]
\end{aligned}
$$

and

$$
\left\langle x x^{\prime}\right\rangle=-\epsilon_{i}\left[A \alpha_{m}+\left(B_{1} \alpha_{m}-B_{2}\right) C+\left(B_{2} \alpha_{m}+B_{1}\right) S\right]
$$


Using Eqs. (7)-(9), and noting that $A, B_{1}$, and $B_{2}$ satisfy $B_{1}^{2}+B_{2}^{2}=A^{2}-1$, we can obtain an analytical expression of emittance growth for arbitrary $f_{\phi}(\phi)$ with small energy spread

$$
\begin{aligned}
\epsilon & =\sqrt{\left\langle x^{2}\right\rangle\left\langle x^{\prime 2}\right\rangle-\left\langle x x^{\prime}\right\rangle^{2}} \\
& =\epsilon_{i} \sqrt{A^{2}-\left(A^{2}-1\right)\left(C^{2}+S^{2}\right)}
\end{aligned}
$$

denote the average relativistic factor of the beam as $\bar{\gamma}$. When the relative energy spread of the beam is very small (i.e., for every particle $|\Delta \gamma|=|\gamma-\bar{\gamma}| \ll \bar{\gamma}$ ), the particle's phase advance in the plasma $\phi$ will become $\phi(\gamma)=$ $\bar{\phi}-\frac{\bar{\phi}}{2 \bar{\gamma}} \Delta \gamma$, where $\bar{\phi}=\phi(\bar{\gamma})$ (See Appendix C for details). Assuming a Gaussian energy distribution, for the beam particles we have:

$$
f_{\gamma}(\gamma)=\frac{1}{\sqrt{2 \pi} \sigma_{\gamma}} \exp \left[-\frac{(\gamma-\bar{\gamma})^{2}}{2 \sigma_{\gamma}^{2}}\right]
$$

As a result, $\phi$ will also have a Gaussian distribution

$$
f_{\phi}(\phi)=\frac{1}{\sqrt{2 \pi} \sigma_{\phi}} \exp \left[-\frac{(\phi-\bar{\phi})^{2}}{2 \sigma_{\phi}^{2}}\right]
$$

where

$$
\sigma_{\phi}=\frac{\bar{\phi}}{2} \frac{\sigma_{\gamma}}{\bar{\gamma}}
$$

$\frac{\sigma_{\gamma}}{\bar{\gamma}}$ is the relative energy spread of the beam. With this Gaussian distribution of $\phi$, we can obtain:

$$
C=\exp \left(-2 \sigma_{\phi}^{2}\right) \cos (2 \bar{\phi}), \quad S=\exp \left(-2 \sigma_{\phi}^{2}\right) \sin (2 \bar{\phi})
$$

Inserting (12) into (10) and using (11), we get an analytical expression of emittance growth for a beam that has a Gaussian energy distribution with a small energy spread:

$$
\frac{\epsilon}{\epsilon_{i}}=A \sqrt{1-\frac{A^{2}-1}{A^{2}} \exp \left[-\left(\frac{\sigma_{\gamma}}{\bar{\gamma}} \bar{\phi}\right)^{2}\right]}
$$

where $\frac{\sigma_{\gamma}}{\bar{\gamma}}$ is the energy spread, and $\bar{\phi}=\frac{1}{\sqrt{2 \bar{\gamma}} c} \int_{0}^{z} \omega_{p}(s) d s$ is the phase change of an electron with energy $\bar{\gamma}$ after it propagates for a longitudinal distance of $z$ inside the plasma. Because we assume the beam's energy, $\bar{\gamma}$, does not change, then

$$
\frac{\epsilon}{\epsilon_{i}}=\frac{\bar{\gamma} \epsilon}{\bar{\gamma} \epsilon_{i}} \approx \frac{\epsilon_{n}}{\epsilon_{n i}}
$$

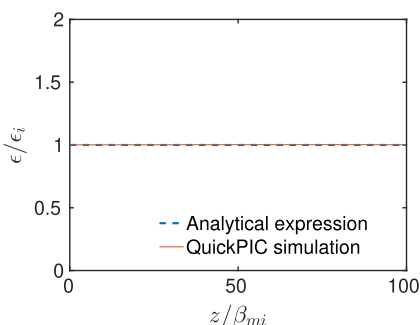

(a)

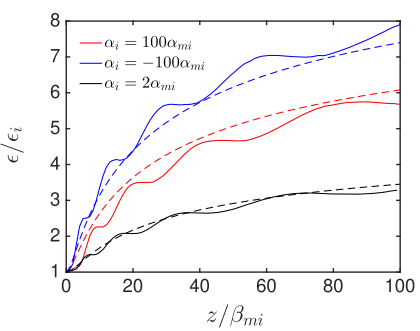

(c)

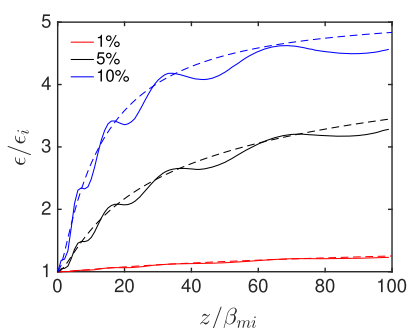

(b)

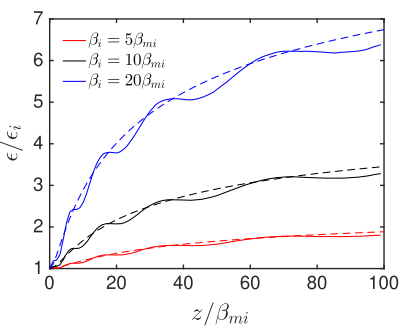

(d)
FIG. 2. Emittance evolution in plasma ramp: $n_{p}(z)=\frac{n_{p 0}}{(1+z / l)^{2}}$ ( $l=5, l$ and $z$ are normalized to $\beta_{m i}$ ). For (a) the beam is initially matched: $\beta_{i}=\beta_{m i}, \alpha_{i}=\alpha_{m i}=-\frac{1}{2 l}=-0.1$, and the beam has a $5 \%$ energy spread. For (b) the beam is initially unmatched: $\beta_{i}=10 \beta_{m i}, \alpha_{i}=2 \alpha_{m i}$, and the beam has $1 \%, 5 \%, 10 \%$ initial energy spreads respectively. For (c) the beam is initially unmatched: $\beta_{i}=10 \beta_{m i}, \alpha_{i}=2 \alpha_{m i}, 100 \alpha_{m i},-100 \alpha_{m i}$ respectively, and the beam has a $5 \%$ energy spread. For (d) the beam is initially unmatched: $\alpha_{i}=2 \alpha_{m i}, \beta_{i}=5 \beta_{m i}, 10 \beta_{m i}, 20 \beta_{m i}$ respectively, and the beam has a $5 \%$ energy spread. In (b)(c)(d), the solid lines are from QuickPIC simulations, and the dashed lines are from the analytical expression (13). In these three plots, the solid black lines correspond to the same simulation result, and the dashed black lines correspond to the same analytical expression.

where $\epsilon_{n}=\frac{1}{m_{e} c} \sqrt{\left\langle x^{2}\right\rangle\left\langle p_{x}^{2}\right\rangle-\left\langle x p_{x}\right\rangle^{2}}$ is the normalized emittance and $p_{x}$ is the transverse momentum of the particle. This means the normalized emittance growth is approximately the same as the geometric emittance growth. Note that in order to keep the analysis tractable we have only kept the effects of the energy spread in the betatron phase advance and not on the amplitude of the betatron oscillation in the elements of the transport matrix. The amplitudes are functions of the local values of the Twiss parameters while the phase is an integral in $z$ over $1 / \beta_{m}$. Therefore, only the phase terms can deviate substantially between particles with small energy differences. Thus, the small amplitude oscillation of the emittance seen in Ref. [13] when a matched beam has finite energy spread is absent here.

In Fig. 2, we compare the theoretical results from (13) with QuickPIC $[17,18]$ simulation results. We choose a plasma density profile $n_{p}(z)=\frac{n_{p 0}}{(1+z / l)^{2}}$, for which the adiabatic condition is independent of $z$. In the simulation, we turn off the longitudinal acceleration for the beam particles (i.e., the energy of the beam particle essentially 
does not change), and choose reasonable parameters to make the simulation in the blowout regime. Figure 2(a) shows that when the beam is initially matched, the beam's emittance is a constant during its propagation inside the plasma. As shown in Fig. 2(b)-2(d), if the beam is not initially matched, the theoretical results based on the WKB solution agree with the simulation result very well.

Note that in (13), $A \geq 1$ is always true (see the Appendix B). So $\epsilon / \epsilon_{i} \leq A$. When the beam propagates in the plasma for a very long distance, $\bar{\phi}$ will become very large, and the beam will have a saturated emittance:

$$
\frac{\epsilon_{\mathrm{sat}}}{\epsilon_{i}}=A=\frac{\beta_{i} \gamma_{m i}+\gamma_{i} \beta_{m i}-2 \alpha_{i} \alpha_{m i}}{2}
$$

For the special case when the plasma is uniform along $z$, we have $\alpha_{m}=\alpha_{m i}=0$, so $\gamma_{m}=\gamma_{m i}=1 / \beta_{m i}$, then

$$
A=\frac{\gamma_{i} \beta_{m i}+\beta_{i} / \beta_{m i}}{2}
$$

Therefore, the emittance growth in a longitudinally uniform plasma will be

$$
\begin{aligned}
\frac{\epsilon}{\epsilon_{i}}= & \frac{\gamma_{i} \beta_{m i}+\beta_{i} / \beta_{m i}}{2} \\
& \times \sqrt{1-\frac{\left(\gamma_{i} \beta_{m i}+\beta_{i} / \beta_{m i}\right)^{2}-4}{\left(\gamma_{i} \beta_{m i}+\beta_{i} / \beta_{m i}\right)^{2}} \exp \left[-\left(\frac{\sigma_{\gamma}}{\bar{\gamma}} \bar{\phi}\right)^{2}\right]}
\end{aligned}
$$

which is mathematically equivalent to Eq. (7) in [11], and similar to Eq. (1) in [10] [difference is due to the different assumptions for $\left.f_{\phi}(\phi)\right]$.

We define the beam to be initially matched when

$$
\alpha_{i}=\alpha_{m i}, \quad \beta_{i}=\beta_{m i}, \quad \gamma_{i}=\gamma_{m i}
$$

Then we have $A=1, B_{1}=0, B_{2}=0$. Therefore, from Eqs. (7)-(9) and (13), we have $\left\langle x^{2}\right\rangle / \epsilon_{i}=\beta_{m},\left\langle x^{\prime 2}\right\rangle / \epsilon_{i}=$ $\gamma_{m}, \quad-\left\langle x x^{\prime}\right\rangle / \epsilon_{i}=\alpha_{m}$ and $\epsilon=\epsilon_{i}$. So $\beta=\left\langle x^{2}\right\rangle / \epsilon=\beta_{m}$, $\gamma=\left\langle x^{\prime 2}\right\rangle / \epsilon=\gamma_{m i}, \quad \alpha=-\left\langle x x^{\prime}\right\rangle / \epsilon=\alpha_{m}$. Therefore, with an adiabatic plasma density profile, when neglecting the beam's energy change, if the beam is initially matched, the beam's Twiss parameters along $z$ will be $\beta_{m}, \gamma_{m}, \alpha_{m}$, and the beam's geometric emittance will not change. We can therefore interpret $\beta_{m}, \alpha_{m}$ defined in (4) and $\gamma_{m}$ as the matched Twiss parameters.

If the beam's initial Twiss parameters deviate from the matched ones, i.e.,

$$
\alpha_{i}=\alpha_{m i}+\Delta \alpha, \quad \beta_{i}=\beta_{m i}+\Delta \beta
$$

where $|\Delta \alpha| \ll 1$ and $|\Delta \beta| / \beta_{m i} \ll 1$, then

$$
A \approx 1+\frac{1}{2} \Delta \alpha^{2}+\frac{\gamma_{m i}}{2 \beta_{m i}} \Delta \beta^{2}-\frac{\alpha_{m i}}{\beta_{m i}} \Delta \alpha \Delta \beta
$$

Inserting this into (13) gives

$$
\frac{\epsilon}{\epsilon_{i}} \approx 1+\left(\frac{1}{2} \Delta \alpha^{2}+\frac{\gamma_{m i}}{2 \beta_{m i}} \Delta \beta^{2}-\frac{\alpha_{m i}}{\beta_{m i}} \Delta \alpha \Delta \beta\right)\left[1-e^{-\left(\frac{\sigma_{Y}}{\bar{\gamma}} \bar{\phi}\right)^{2}}\right]
$$

We can also get the expression for $\beta$ from the above equations. Inserting (12) into (7) and using (11) leads to

$$
\left\langle x^{2}\right\rangle=\epsilon_{i} \beta_{m}\left\{A+\left[B_{1} \cos (2 \bar{\phi})+B_{2} \sin (2 \bar{\phi})\right] e^{-\frac{1}{2}\left(\frac{\sigma_{\bar{\gamma}}}{\bar{\gamma}} \bar{\phi}\right)^{2}}\right\} .
$$

Dividing both sides by $\epsilon$ and using (13) gives

$$
\beta=\beta_{m} \frac{A+\left[B_{1} \cos (2 \bar{\phi})+B_{2} \sin (2 \bar{\phi})\right] \exp \left[-\frac{1}{2}\left(\frac{\sigma_{\gamma}}{\bar{\gamma}} \bar{\phi}\right)^{2}\right]}{A \sqrt{1-\frac{A^{2}-1}{A^{2}} \exp \left[-\left(\frac{\sigma_{\gamma}}{\bar{\gamma}} \bar{\phi}\right)^{2}\right]}} .
$$

If there is no energy spread $\left(\sigma_{\gamma}=0\right)$, this equation can be simplified

$$
\beta=\beta_{m}\left[A+B_{1} \cos (2 \phi)+B_{2} \sin (2 \phi)\right]
$$

which is similar in form to Eq. (11) in [13] but with different coefficients.

\section{ON MINIMIZING THE EMITTANCE GROWTH FOR A FIXED BEAM}

In the previous section, it was shown that the beam emittance will be preserved as long as the beam satisfies the matching condition [Eq. (16)]. In this case $\beta^{*}=1 / \gamma_{m i}\left(\beta^{*}\right.$ is $\beta$ when $\alpha=0$ ) which we define as the matched $\beta^{*}, \beta_{m}^{*}$. It was also shown how the emittance grows if the beam is slightly mismatched as might be the case if there are shot to shot variations of the beam and/or plasma conditions. In addition, in a controlled experiment that might for example be conducted at FACET II [14], the beam emittance and optics are relatively fixed so that $\beta^{*}$ can be assume to be fixed. However, for a given plasma profile the plasma conditions at the plasma entrance are such that it will not be possible to match a beam with a given $\beta^{*}$ (i.e., $\beta^{*} \neq \beta_{m}^{*}$ ). It is therefore useful to determine the best location to focus such a beam. This is defined to be the focal position in vacuum $(z=s)$ that minimizes the emittance growth for a given beam and plasma profile, assuming the plasma entrance is at $z=0$.

Therefore,

$$
\alpha(s)=0, \quad \beta(s)=\beta^{*}
$$

where $\beta^{*}$ is $\beta$ at the focal plane. According to the evolution of Twiss parameters in a drift space [19], the beam's initial Twiss parameters at the plasma entrance $(z=0)$ are 
$\alpha_{i}=\alpha(0)=\frac{s}{\beta^{*}}, \quad \beta_{i}=\beta(0)=\beta^{*}+\frac{s^{2}}{\beta^{*}}, \quad \gamma_{i}=\gamma(0)=\frac{1}{\beta^{*}}$

Using Eq. (13), we can calculate the emittance growth using the above initial condition, and find the optimal $s$ defined to be when $d \epsilon / d s=0$. For a fixed plasma density profile, $d \epsilon / d s=0$ reduces to $d A / d s=0$, which gives us the optimal $s$,

$$
s_{o}=\frac{\alpha_{m i}}{\gamma_{m i}}
$$

This optimal $s=s_{o}$ is actually the focal position in vacuum for the matched beam (whose initial Twiss parameters at the plasma entrance are $\alpha_{m i}, \beta_{m i}$ and $\gamma_{m i}$ ). In other words, by putting the unmatched beam's focal plane at the same position as the matched beam's focal plane in vacuum, the unmatched beam will have minimal emittance growth in the plasma. We can calculate this minimal emittance growth by evaluating $A$ using the initial Twiss parameters from (20) and (21), giving $A=A_{o} \equiv \frac{1}{2}\left(\beta^{*} \gamma_{m i}+\frac{1}{\beta^{*} \gamma_{m i}}\right)$. We then insert this into (13) to get the minimal emittance growth.

If the witness beam's focal plane in vacuum deviates from the optimal position (21): $s=s_{o}+\Delta s$, from (20) and (21) we can obtain:

$$
A=A_{o}+\frac{\gamma_{m i}}{2 \beta^{*}} \Delta s^{2}
$$

We can see that for $s=s_{o}+\Delta s$ and $s=s_{o}-\Delta s$, the corresponding $A$ are the same, so according to (13), the emittance growth are the same as well. In other words, the emittance growth as a function of $s$ is symmetric about $s=s_{o}$.

If we assume $\Delta s$ is a small quantity, then for a fixed $z$ we get,

$$
\frac{\epsilon}{\epsilon_{i}} \approx \frac{\epsilon_{o}}{\epsilon_{i}}+\left\{1-\exp \left[-\left(\frac{\sigma_{\gamma}}{\bar{\gamma}} \bar{\phi}\right)^{2}\right]\right\} \frac{A_{o}}{\epsilon_{o} / \epsilon_{i}} \frac{\gamma_{m i}}{2 \beta^{*}} \Delta s^{2}
$$

where $\epsilon_{o} \equiv \epsilon\left(A_{o}\right) \quad$ is the emittance when $\Delta s=0$ (or $s=s_{o}$ ).

This analysis also permits examining how the shot to shot variance of the plasma density at the entrance of the profile affects the emittance growth (essentially $A$ ), assuming the beam profile and the position of the plasma entrance $\left(\beta^{*}\right.$ and $\left.s\right)$ are fixed. From Eq. (14) and the relation $\gamma_{m i}=\left(1+\alpha_{m i}^{2}\right) / \beta_{m i}$, we have

$$
A=\frac{\beta_{i}\left(1+\alpha_{m i}^{2}\right) / \beta_{m i}+\gamma_{i} \beta_{m i}-2 \alpha_{i} \alpha_{m i}}{2}
$$

Since we assume the beam profile and the position of the plasma entrance are fixed, from Eq. (20) we know $\alpha_{i}, \beta_{i}, \gamma_{i}$ are fixed. The shot to shot changes to the plasma profile lead to the variances of $\alpha_{m i}$ and $\beta_{m i}$, which leads to the first order variance of $A$ :

$$
\begin{aligned}
\delta A_{1} & =\frac{\partial A}{\partial \alpha_{m i}} \delta \alpha_{m i}+\frac{\partial A}{\partial \beta_{m i}} \delta \beta_{m i} \\
& =\left(\frac{\beta_{i}}{\beta_{m i}} \alpha_{m i}-\alpha_{i}\right) \delta \alpha_{m i}+\frac{1}{2}\left(\gamma_{i}-\gamma_{m i} \frac{\beta_{i}}{\beta_{m i}}\right) \delta \beta_{m i}
\end{aligned}
$$

Especially, we can see that at the matching point, the variance of $\alpha_{m i}$ and $\beta_{m i}$ will not cause the variance of $A$ to the first order. The second order variation of $A$ is

$$
\delta A_{2}=\frac{1}{2} \delta \alpha_{m i}^{2}+\frac{\gamma_{m i}}{2 \beta_{m i}} \delta \beta_{m i}^{2}-\frac{\alpha_{m i}}{\beta_{m i}} \delta \alpha_{m i} \delta \beta_{m i}
$$

which is consistent with equation (17). Essentially, variations can arise from either the plasma profile or beam profile at the plasma entrance.

Next we carry out some QuickPIC simulations using plasma and beam parameters that are close to the ones in the proposed FACET II experiment, while satisfying all the theoretical assumptions (adiabatic plasma profile, azimuthal symmetry in plasma wake, etc). We turn off the longitudinal push in the simulation so that the beam has no longitudinal acceleration. The plasma density profile we use is shown in Fig. 3(b). This profile is the region between $5 \mathrm{~cm}$ and $75 \mathrm{~cm}$ of the full profile [Fig. 3(a)]. The adiabatic condition $\left(\left|\alpha_{m}\right|<1\right)$ is now satisfied throughout the entire profile. At the entrance and exit $\left|\alpha_{m}\right|=0.24$ and 0.56 respectively. In addition, $\alpha_{m i}=0.24, \beta_{m i}=0.0194 \mathrm{~m}$ for the simulation. The theory could be easily modified to include a matching section [10] or a perturbative section as in [13]. We use a nonevolving symmetric drive beam to create a well formed ion bubble, and the witness beam is the same as the one in FACET II (See Tables I and II). Figure 4 shows the simulation results and the good

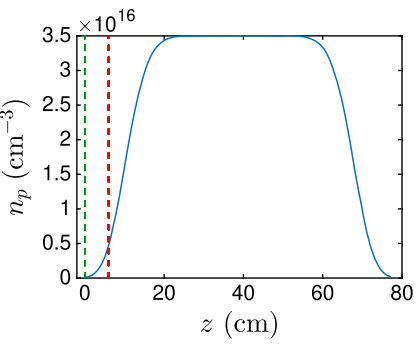

(a)

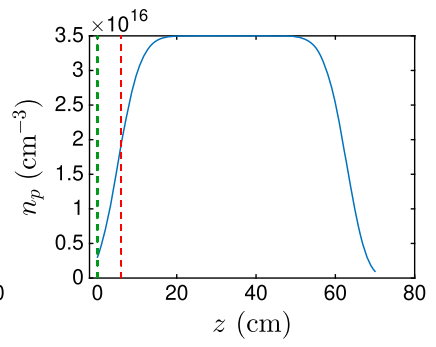

(b)
FIG. 3. Plasma density profile. The green dashed line is the entrance of the plasma, and the red dashed line is the position of the witness beam's focal plane in vacuum. The beams propagate to the right in the plot. (a) The FACET II plasma density profile. (b) The profile used for the simulation results in Fig. 4. Only the region between $5 \mathrm{~cm}$ and $75 \mathrm{~cm}$ of the profile in (a) is used. In this region the adiabatic condition is always satisfied. 


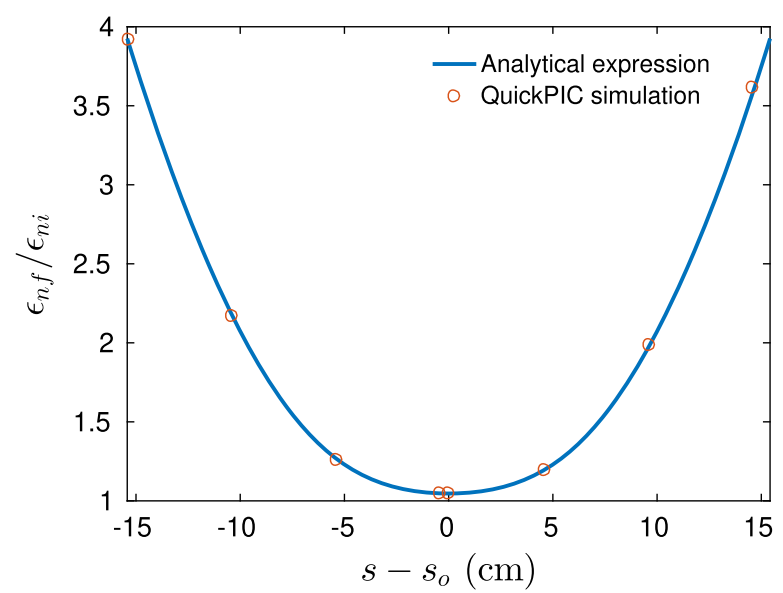

(a)

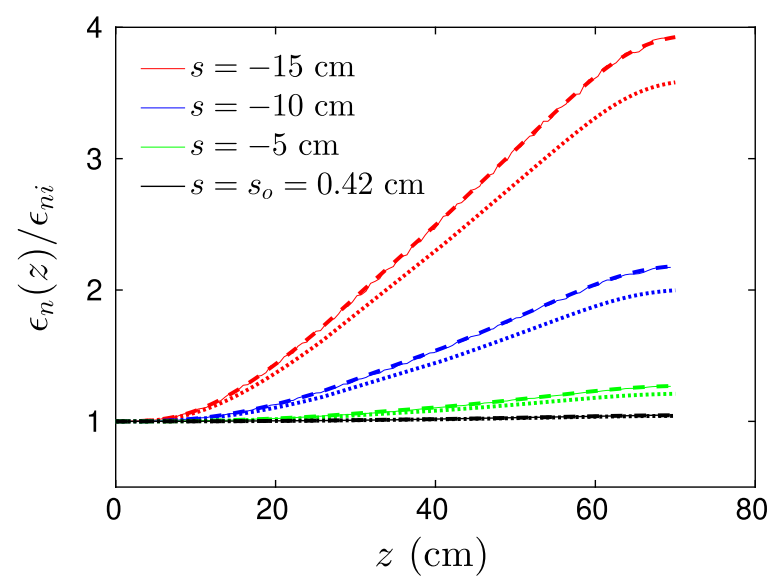

(b)

FIG. 4. Witness beam's emittance growth for different focal planes, $s$, in the adiabatic plasma in Fig. 3(b). (a) The ratio of final emittance (at the plasma exit) to the initial emittance (at the plasma entrance) for different cases. (b) The evolution of $\epsilon_{n}$ inside the plasma for 4 different cases, corresponding to the 4 orange spots for $s-s_{o} \leq 0$ in (a). The solid lines are from QuickPIC simulations, the dashed lines are from expression (13), and the dotted lines are from expression (15).

agreement between the simulations and the theory. It also shows that the expression for emittance growth in a uniform plasma [Eq. (15)] cannot describe the emittance growth in an adiabatic plasma precisely.

\section{EMITTANCE EVOLUTION IN PREFORMED PLASMA USING FACET II PARAMETERS}

Table I shows a possible set of beams parameters for the two-bunch FACET II experiments.

Both beams are tri-Gaussian with $n_{b}=\frac{N}{(2 \pi)^{\frac{3}{2}}} e^{-\frac{x^{2}}{2 \sigma_{x}^{2}}} e^{-\frac{y^{2}}{2 \sigma_{y}^{2}}}$ $e^{-\frac{z^{2}}{2 \sigma_{z}^{2}}}$. The $\sigma_{z}$ is the rms pulse length for the beam, and $\epsilon_{n_{x}}$ and $\epsilon_{n_{y}}$ are the normalized emittance in $x, y$ directions
TABLE I. Possible beam parameters for two-bunch PWFA experiment at FACET II.

\begin{tabular}{lccccc}
\hline \hline & Energy $(\mathrm{GeV})$ & $\mathrm{Q}(\mathrm{nC})$ & $\sigma_{z}(\mu \mathrm{m})$ & $\epsilon_{n_{x}}(\mu \mathrm{m})$ & $\epsilon_{n_{y}}(\mu \mathrm{m})$ \\
\hline Drive & 10 & 1.6 & 6.4 & 3.4 & 3.0 \\
Witness & 10 & 0.5 & 5.0 & 3.15 & 3.15 \\
\hline \hline
\end{tabular}

respectively. The distance between the drive and witness beam is $150 \mu \mathrm{m}$. The initial relative rms energy spread for both beams is $0.25 \%$. Table II shows the Twiss parameters for both beams at the exit of the final focusing magnet. Note that in this setup the drive beam is asymmetric and the witness beam is symmetric, so the wake felt by the witness beam is not azimuthally symmetric.

The plasma density profile in the simulation is shown in the Fig. 3(a), which is close to the plasma density profile of the lithium source used in the FACET II experiment. The peak plasma density is $3.5 \times 10^{16} \mathrm{~cm}^{-3}$, which is chosen to ensure that the witness beam is located inside the bubble wake at a position that flattens the accelerating field (as shown in Fig. 1).

With such a plasma density profile, the initial matched Twiss parameters for the witness beam at the plasma entrance are:

$$
\alpha_{m i}=0.916, \quad \beta_{m i}=0.068 \mathrm{~m}
$$

These parameters are not calculated directly from (4) at $z=0$ because the plasma near the entrance does not satisfy the adiabatic condition (5). Instead, they are obtained by neglecting any energy spread, and integrating the following equation (See Appendix D for derivation) for $\beta$,

$$
\begin{aligned}
\frac{1}{2} \beta(z) \beta^{\prime \prime}(z)-\frac{1}{4} \beta^{\prime}(z)^{2}+\beta(z)^{2} k_{\beta}(z)^{2} & =1 \\
\alpha(z) & =-\frac{1}{2} \beta^{\prime}(z)
\end{aligned}
$$

from the flat-topped region of the plasma back to the entrance of the plasma with initial Twiss parameters $\beta=\sqrt{2 \bar{\gamma}} \frac{c}{\omega_{p}}, \alpha=0$ (where $\omega_{p}$ is the plasma frequency for the flat-topped plasma) [14]. According to the matched parameters given in (23), the optimal $s$ for the plasma density profile can be calculated from (21),

TABLE II. The Twiss parameters of both beams at the exit of the final focusing magnet.

\begin{tabular}{lcccc}
\hline \hline & $\alpha_{x}$ & $\alpha_{y}$ & $\beta_{x}(m)$ & $\beta_{y}(m)$ \\
\hline Drive Beam & 59 & 12 & 127 & 27 \\
Witness Beam & 40 & 40 & 80 & 80 \\
\hline \hline
\end{tabular}




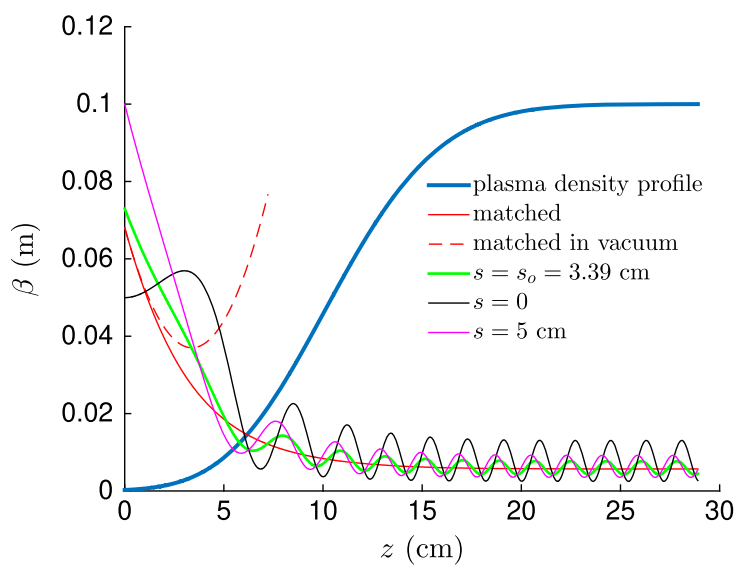

FIG. 5. The evolution of $\beta$ for the witness beam for different $s$ from numerical calculation. The plasma density profile is in arbitrary units.

$s=s_{o}=3.39 \mathrm{~cm}$. Figure 5 shows the evolution of $\beta$ for the real witness beam when its focal plane in vacuum is located at a different $s=s_{o}+\Delta s$. The solid red line is the case if the witness beam was initially matched to the plasma profile. We can see that for a matched beam $\beta$ evolves smoothly and stays constant in the uniform plasma region while for an unmatched beam the beam's $\beta$ will oscillate.

Next, we run QuickPIC simulations in which we vary $s$ but with the same beams as described in Tables I and II. This time we turn on the longitudinal push in the simulation so the witness beam is gaining energy. Figure 6(a) shows the normalized emittance growth at the exit of the plasma when the witness beam's focal plane in vacuum is located at $s=-10,-5,0,3.39,5,10$, $15,20 \mathrm{~cm}$ (note that negative $s$ means the focal plane of the witness beam is outside the plasma). We can clearly see that the optimal $s$ for minimizing the emittance growth is at $s=3.39 \mathrm{~cm}$. This illustrates that experiments can be performed at FACET II that provide easily measurable differences in the emittance growth as the focal point is changed.

We note that the different emittance growth in $x$ and $y$ directions is caused by the asymmetry of the drive beam, which excites asymmetric wakefields that have different linear focusing forces in $x$ and $y$ directions [20]. We note that even though the plasma and the beam parameters used in these simulations do not satisfy the assumptions we made in the previous sections (the drive beam is asymmetric and the plasma near the entrance and the exit is not adiabatic), (21) still appears to predict the optimal focal position of the witness beam very well, although the initial matched Twiss parameters $\alpha_{m i}, \beta_{m i}$ are calculated in a different way. Figure 6(b) shows the evolution of witness beam's $\epsilon_{n_{x}}$ along $z$. We can see that when $s=3.39 \mathrm{~cm}, \epsilon_{n_{x}}$ is almost preserved although the beam is not initially matched.

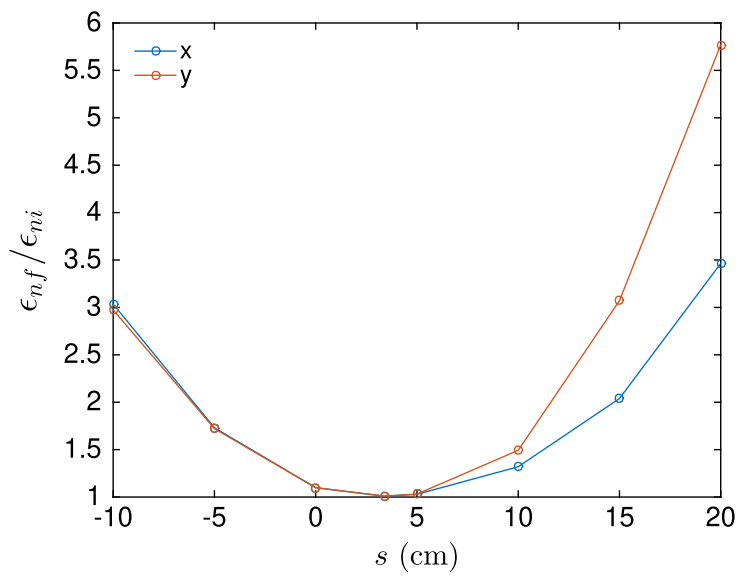

(a)

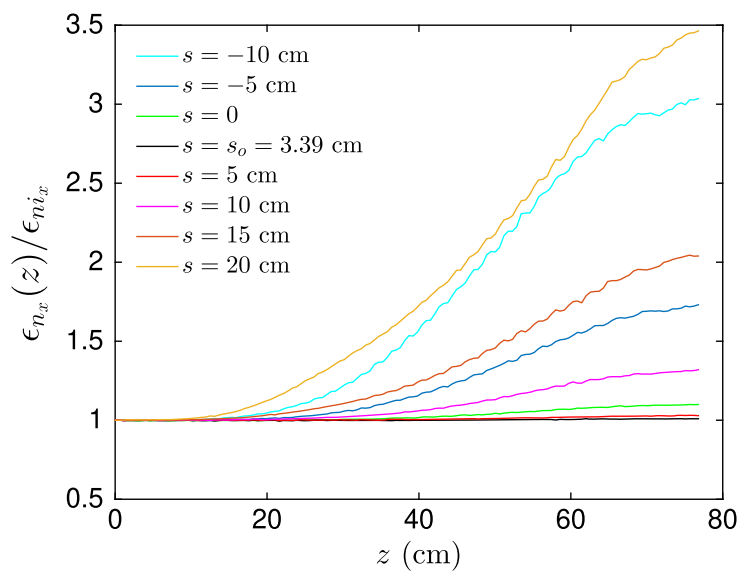

(b)

FIG. 6. The normalized emittance growth of the witness beam for different $s$. (a) The ratio of final emittance (at the plasma exit) to the initial emittance (at the plasma entrance) for different cases. (b) The evolution of $\epsilon_{n_{x}}$ inside the plasma for different cases, corresponding to the blue line in (a).

\section{EMITTANCE EVOLUTION IN LITHIUM PLASMA WITH HELIUM BUFFER GAS}

In FACET II experiments, lithium will be one of the choices for the plasma source. The hot lithium vapor will be confined and cooled at each end by the helium buffer gas $[1,2]$. The plasma is generated by the intense electric field of the drive/witness beams when they pass through and ionize the lithium vapor. In the previous section, we simulated the beams evolving in a preformed and radially uniform plasma. In this section, we use QuickPIC to simulate the emittance evolution when the plasma is self-formed by field ionization of a neutral gas from the intense electric field of the drive and witness beams. Figure 7 shows the profile for the lithium gas and the helium buffer gas in our simulation.

The blue line in Fig. 7 is the lithium gas density, which is the same as the profile shown in Fig. 3(a) in the previous section. There are two linear helium ramps (orange line 


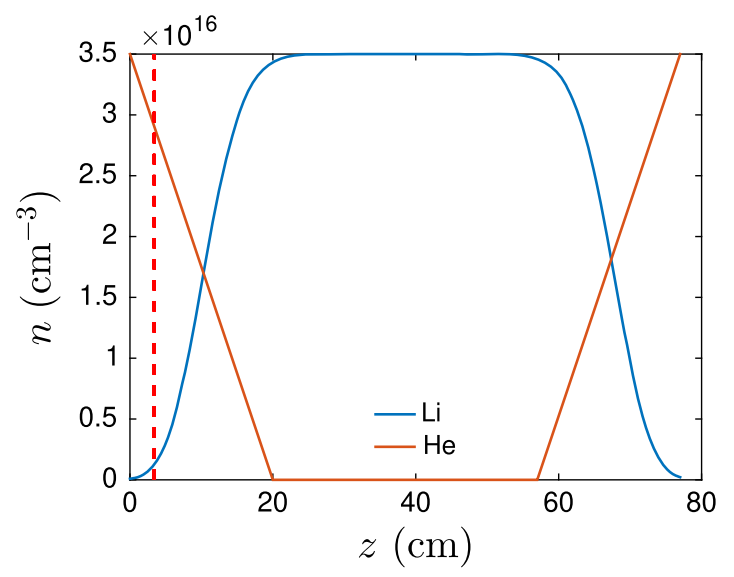

FIG. 7. Helium and lithium gas density profile. The red dashed line is the position of the witness beam's focal plane: $z=3.39 \mathrm{~cm}$.

in Fig. 7) at the entrance and exit of the lithium gas. The beam parameters are the same as described in the previous section, and we choose the optimal value $s=3.39 \mathrm{~cm}$ for the witness beam's focal position in vacuum. Figure 8(a) shows the witness beam's emittance evolution inside the plasma. We can see that in the beginning and the end of the simulation, emittance growth occurs. In the middle of the lithium region where there is no helium, the emittance essentially stays the same.

The reason for the large emittance growth is that the beams can ionize the helium buffer gas which results in a nonlinear focusing force inside the bubble. Figure 9(a) shows the helium ion density snapshot when the beams propagate for $1 \mathrm{~cm}$ in the plasma. The drive beam's center is located at $\xi=-5.27$, and the witness beam's center is located at $\xi=0$. Both beams are propagating from right to left. The drive beam enters the plasma with a larger spot size than the witness beam, so it can only ionize a part of the neutral helium while the witness beam can ionize most of the neutral helium around it. Figure 9(b) shows the

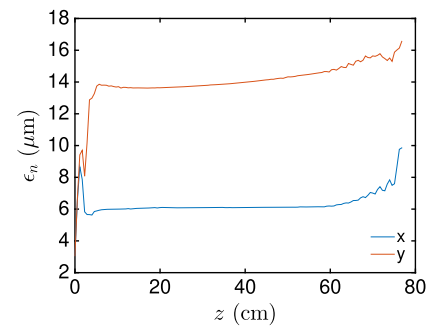

(a)

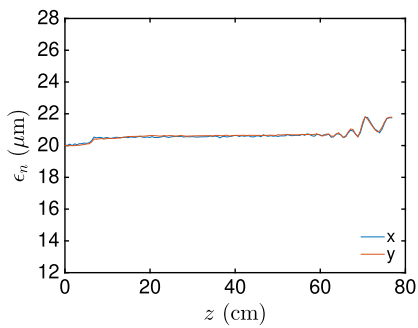

(b)
FIG. 8. The evolution of normalized emittance of the witness beam: (a) We use the same parameters as we used in the preformed plasma simulation in the previous section: Drive beam: $\epsilon_{n_{x}}=3.4 \mu \mathrm{m}, \epsilon_{n_{y}}=3.0 \mu \mathrm{m}$, witness beam: $\epsilon_{n_{x}}=\epsilon_{n_{y}}=$ $3.15 \mu \mathrm{m}$. (b) We increase the initial emittance for both beams to $20 \mu \mathrm{m}$ (in both $x$ and $y$ directions).

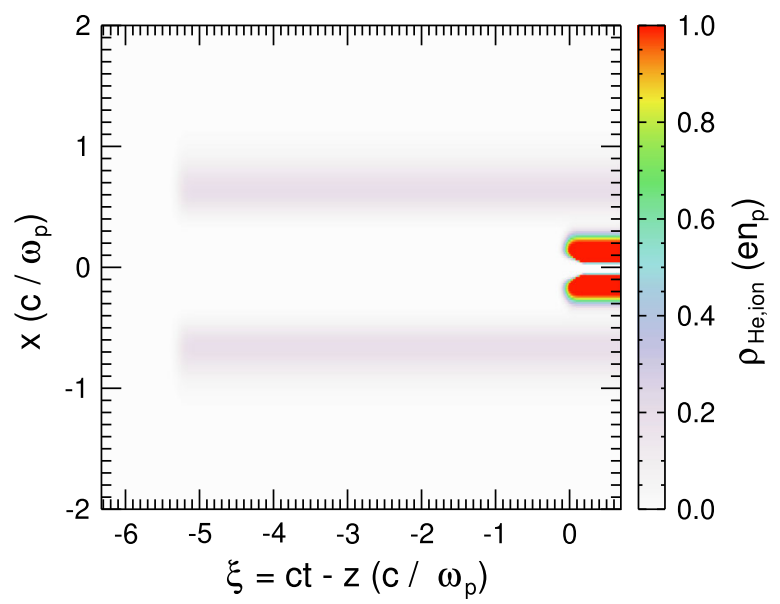

(a)

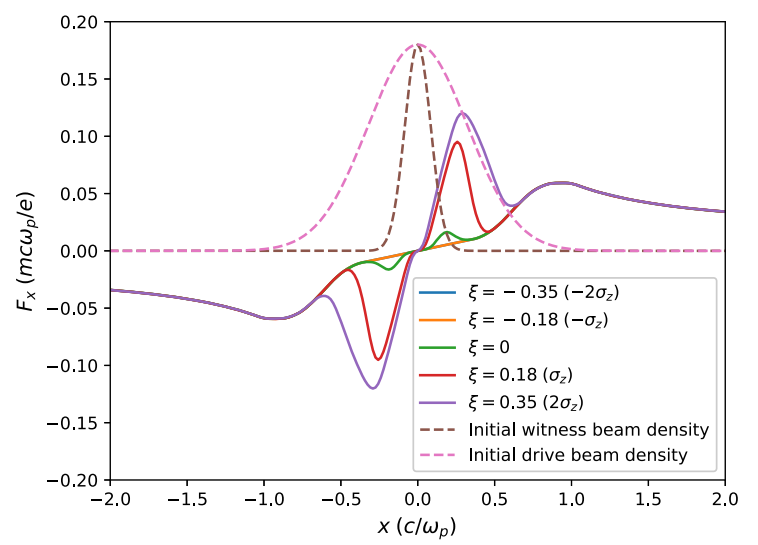

(b)

FIG. 9. (a) Helium ion charge density $\rho_{\mathrm{He} \text {,ion. The grey area is }}$ the helium ions produced by the drive beam, and the red area is the helium ions produced by the witness beam. (b) The $F_{x}$ transverse lineouts at different longitudinal positions, $\xi$, and the initial beam density profiles (in arbitrary units).

focusing fields felt by the witness beam at different $\xi$ at the same propagation distance as Fig. 9(a). The focusing fields felt by the witness beam is no longer linear when helium is ionized by itself. Therefore, the witness beam has a large emittance growth in the region where the helium gas is ionized. In the region where there is only lithium, the witness beam will still feel a linear focusing force and its emittance only grows because the witness beam is no longer matched in the uniform region of lithium plasma, which causes a much smaller growth than that from the region where helium gas exists.

In order to avoid the emittance growth in the lithium plasma source, we can increase the initial emittances for both the drive beam and witness beam. In Fig. 8(b), we show the QuickPIC simulation results when using an initial beam emittance of $20 \mu \mathrm{m}$ while keeping the other parameters the same as the simulation shown in Fig. 8(a). When the initial beam emittance becomes larger, the initial spot sizes of both beams will increase, and the Coulomb field 
around the beam will become smaller. Therefore, when the beams pass through the helium buffer gas, the neutral helium is weakly ionized. However, the lithium can still be ionized and form the plasma wake because lithium has a lower ionization energy than helium. When there is no helium ionization, the focusing force felt by the witness beam is linear, and its emittance barely grows, as shown in Fig. 8(b). The small emittance growth at the exit of the plasma in Fig. 8(b) is still caused by the helium ionization because the witness beam enters into the exit ramp of helium with a smaller spot size compared to its initial spot size at the entrance of the plasma.

\section{CONCLUSION}

We have used theory and QuickPIC simulations to examine the evolution of the emittance and the Twiss parameters of particle beams in plasmas whose density is changing adiabatically. We use the WKB solution for each particle and assume the energy of each particle in the beam does not change to obtain an analytical expression for the beam's emittance evolution in an arbitrary adiabatic plasma density profile in a nonlinear PWFA. When the beam has no initial energy spread, its emittance will remain a constant in the azimuthally symmetric blowout regime. When there is an initial energy spread, the beam's emittance can be preserved as long as its initial Twiss parameters are matched to the density profile of the plasma ramp. We also use this expression to analyze the emittance growth when the position of the witness beam's focal plane in vacuum is changed while keeping the beam parameters and the plasma density profile fixed. When the beam cannot be matched, the emittance growth can be minimized by focusing the unmatched beam to the same vacuum focal plane position as the matched beam. We used QuickPIC simulations for possible FACET II beam parameters to show that the emittance can indeed be preserved very well when we choose the focal plane position to be the same as that for a matched beam, even when the assumptions of symmetric blowout and adiabatic density evolution for the entire plasma region are not satisfied. For other focal plane positions, the witness beam's emittance is larger at the exit of the plasma.

In addition, we also examined through simulations the effect of additional self-ionization of the buffer gas by the drive beam. At FACET II a lithium gas is confined by a helium buffer gas. When the drive and/or witness beam emittance is small (around $3 \mu \mathrm{m}$ ), they can be focused to small enough spot sizes so that they can ionize the helium buffer gas. This will lead to the focusing fields felt by the witness beam to be strongly nonlinear. We find that this can potentially lead to the witness beam's emittance growing by a factor of 3 and 5 in the $x$ and $y$ planes respectively for sample FACET II parameters. The different growth in $x$ and $y$ directions is caused by the asymmetry of the drive beam forming an asymmetric plasma wake. By using an initial emittance of $20 \mu \mathrm{m}$, the helium buffer gas is weakly ionized and the witness beam's emittance can be preserved very well.

\section{ACKNOWLEDGMENTS}

This work was supported by the U.S. Department of Energy under Grants No. DE-SC0010064, and SCIDAC under Grants No. 644405, and the National Science Foundation under Grants No. 1806046. The simulations were run on Bluewaters, NERSC, and the UCLA Hoffman2 clusters.

\section{APPENDIX A: CALCULATION OF BEAM MOMENT $\left\langle x^{2}\right\rangle$}

In this Appendix we provide details on calculating the second moment of the beam, i.e., the square of the spotsize.

$$
\begin{aligned}
\left\langle x^{2}\right\rangle & =\int x^{2} f\left(x, x^{\prime}\right) d x d x^{\prime} \\
& =\int x^{2} f_{i}\left(x_{i}, x_{i}^{\prime}\right) d x_{i} d x_{i}^{\prime} \\
& =\int\left(M_{11} x_{i}+M_{12} x_{i}^{\prime}\right)^{2} f_{i}\left(x_{i}, x_{i}^{\prime}\right) d x_{i} d x_{i}^{\prime}
\end{aligned}
$$

where $f\left(x, x^{\prime}\right)$ is the distribution function at $z$ and $f_{i}$ is the initial distribution function. From the Vlasov equation we have $f\left(x, x^{\prime}\right)=f_{i}\left(x_{i}, x_{i}^{\prime}\right)$, and $d x d x^{\prime}=d x_{i} d x_{i}^{\prime}$ because $\operatorname{det}(\mathbf{M})=1$.

The last step above is correct only if all the particles have the same energy. However, since different particles have different energy $\gamma$, their corresponding transport matrices $M$ are different. In order to calculate the above integral with an energy spread in the beam, we assume the main difference in $M$ is the phase advance $\phi$. Even though the $\beta_{m}, \alpha_{m}$ in $M$ are different (because of different $\gamma$ ), we assume them to be the same for all the particles and use $\gamma=\bar{\gamma}(\bar{\gamma}$ is the mean energy among all the particles), while claiming the main difference is in $\phi$ due to different energy $\gamma$. After the beam propagates for a distance $z$, we denote the distribution of the phase advance $\phi$ as $f_{\phi}(\phi)$ (with the normalization $\left.\int f_{\phi}(\phi) d \phi=1\right)$. So:

$$
\begin{aligned}
\left\langle x^{2}\right\rangle= & \iiint\left(M_{11} x_{i}+M_{12} x_{i}^{\prime}\right)^{2} f\left(x_{i}, x_{i}^{\prime}\right) f_{\phi}(\phi) d x_{i} d x_{i}^{\prime} d \phi \\
= & \left\langle x_{i}^{2}\right\rangle \int d \phi f_{\phi}(\phi) M_{11}^{2}+\left\langle x_{i}^{\prime}\right\rangle^{2} \int d \phi f_{\phi}(\phi) M_{12}^{2} \\
& +\left\langle x_{i} x_{i}^{\prime}\right\rangle \int d \phi f_{\phi}(\phi) 2 M_{11} M_{12} \\
= & \epsilon_{i}\left[\beta_{i} \int d \phi f_{\phi}(\phi) M_{11}^{2}+\gamma_{i} \int d \phi f_{\phi}(\phi) M_{12}^{2}\right. \\
& \left.-\alpha_{i} \int d \phi f_{\phi}(\phi) 2 M_{11} M_{12}\right]
\end{aligned}
$$


where

$$
\begin{aligned}
\int d \phi f_{\phi}(\phi) M_{11}^{2} & =\frac{\beta_{m}}{\beta_{m i}} \int d \phi f_{\phi}(\phi)\left(\cos \phi+\alpha_{m i} \sin \phi\right)^{2} \\
& =\frac{1}{2} \frac{\beta_{m}}{\beta_{m i}}\left[(1+C)+\alpha_{m i}^{2}(1-C)+2 \alpha_{m i} S\right] \\
& =\frac{1}{2} \frac{\beta_{m}}{\beta_{m i}}\left[\beta_{m i} \gamma_{m i}+\left(1-\alpha_{m i}^{2}\right) C+2 \alpha_{m i} S\right] \\
\int d \phi f_{\phi}(\phi) M_{12}^{2} & =\beta_{m} \beta_{m i} \int d \phi f_{\phi}(\phi) \sin ^{2} \phi \\
& =\frac{1}{2} \beta_{m} \beta_{m i}(1-C) \\
\int d \phi f_{\phi}(\phi) 2 M_{11} M_{12} & =\beta_{m} \int d \phi f_{\phi}(\phi)\left(2 \cos \phi \sin \phi+2 \alpha_{m i} \sin ^{2} \phi\right) \\
& =\beta_{m}\left[S+\alpha_{m i}(1-C)\right]
\end{aligned}
$$

and where

$$
\begin{aligned}
& C=\int d \phi f_{\phi}(\phi) \cos 2 \phi, \\
& S=\int d \phi f_{\phi}(\phi) \sin 2 \phi
\end{aligned}
$$

Finally, we obtain:

$$
\begin{aligned}
\left\langle x^{2}\right\rangle= & \epsilon_{i} \beta_{m}\left[\frac{\beta_{i} \gamma_{m i}+\gamma_{i} \beta_{m i}-2 \alpha_{i} \alpha_{m i}}{2}\right. \\
& +\left(\frac{\beta_{i}}{\beta_{m i}}-\frac{\beta_{i} \gamma_{m i}+\gamma_{i} \beta_{m i}+2 \alpha_{i} \alpha_{m i}}{2}\right) C \\
& \left.+\left(\frac{\beta_{i}}{\beta_{m i}} \alpha_{m i}-\alpha_{i}\right) S\right]
\end{aligned}
$$

We can define:

$$
\begin{aligned}
A & =\frac{\beta_{i} \gamma_{m i}+\gamma_{i} \beta_{m i}-2 \alpha_{i} \alpha_{m i}}{2} \\
B_{1} & =\frac{\beta_{i}}{\beta_{m i}}-A=\frac{\beta_{i}}{\beta_{m i}}-\frac{\beta_{i} \gamma_{m i}+\gamma_{i} \beta_{m i}-2 \alpha_{i} \alpha_{m i}}{2} \\
B_{2} & =\frac{\beta_{i}}{\beta_{m i}} \alpha_{m i}-\alpha_{i} .
\end{aligned}
$$

Leading to:

$$
\left\langle x^{2}\right\rangle=\epsilon_{i} \beta_{m}\left(A+B_{1} C+B_{2} S\right) .
$$

\section{APPENDIX B: PROOF OF $A \geq 1$}

$$
\begin{aligned}
A & =\frac{\beta_{i} \gamma_{m i}+\gamma_{i} \beta_{m i}-2 \alpha_{i} \alpha_{m i}}{2} \\
& \geq \frac{2 \sqrt{\beta_{i} \gamma_{m i} \gamma_{i} \beta_{m i}}-2 \alpha_{i} \alpha_{m i}}{2} \\
& =\sqrt{\left(1+\alpha_{i}^{2}\right)\left(1+\alpha_{m i}^{2}\right)}-\alpha_{i} \alpha_{m i} \\
& =\sqrt{1+\alpha_{i}^{2}+\alpha_{m i}^{2}+\alpha_{i}^{2} \alpha_{m i}^{2}}-\alpha_{i} \alpha_{m i} \\
& \geq \sqrt{1+2 \alpha_{i} \alpha_{m i}+\alpha_{i}^{2} \alpha_{m i}^{2}}-\alpha_{i} \alpha_{m i} \\
& =\left|1+\alpha_{i} \alpha_{m i}\right|-\alpha_{i} \alpha_{m i} \\
& \geq 1
\end{aligned}
$$

\section{APPENDIX C}

In this Appendix we derive the first order correction to the phase advance due to variation of the energy of the particle. We begin with the definition for the phase

$$
\phi=\int_{0}^{z} \frac{\omega_{p}(s)}{\sqrt{2 \gamma} c} d s
$$

Due to the variation of $\gamma$, the variation of $\phi$ is

$$
\begin{aligned}
\Delta \phi & =\Delta \int_{0}^{z} \frac{\omega_{p}(s)}{\sqrt{2 \gamma} c} d s=\int_{0}^{z} \Delta\left(\frac{\omega_{p}(s)}{\sqrt{2 \gamma} c}\right) d s \\
& =\int_{0}^{z}-\frac{1}{2}\left(\frac{\omega_{p}(s)}{\sqrt{2} \gamma^{\frac{3}{2}} c}\right) \Delta \gamma d s=-\frac{\Delta \gamma}{2 \gamma} \int_{0}^{z} \frac{\omega_{p}(s)}{\sqrt{2 \gamma} c} d s \\
& =-\frac{\phi}{2 \gamma} \Delta \gamma
\end{aligned}
$$


So the difference between the phase advance of a particle with energy $\gamma$ and the phase advance of a particle with the average energy $\bar{\gamma}$ is

$$
\phi(\gamma)-\phi(\bar{\gamma})=-\frac{\phi(\bar{\gamma})}{2 \bar{\gamma}} \Delta \gamma
$$

and

$$
\phi(\gamma)=\bar{\phi}-\frac{\bar{\phi}}{2 \bar{\gamma}} \Delta \gamma
$$

\section{APPENDIX D: DIFFERENTIAL EQUATION FOR $\beta$}

In this Appendix, we offer a derivation of Eq. (24) in the text. We start from the definition of the beam's spot size:

$$
\sigma_{x}=\sqrt{\left\langle x^{2}\right\rangle}
$$

Taking derivatives with respect to $z$ provide:

$$
\begin{aligned}
\sigma_{x}^{\prime} & =\frac{\left\langle x x^{\prime}\right\rangle}{\sigma_{x}} \\
\sigma_{x}^{\prime \prime} & =\frac{\left\langle x^{2}\right\rangle\left\langle x^{\prime 2}\right\rangle-\left\langle x x^{\prime}\right\rangle^{2}}{\sigma_{x}^{3}}+\frac{\left\langle x x^{\prime \prime}\right\rangle}{\sigma_{x}} .
\end{aligned}
$$

Using the definition of geometric emittance (6) and the equation of motion (1), leads to:

$$
\sigma_{x}^{\prime \prime}=\frac{\epsilon^{2}}{\sigma_{x}^{3}}-k_{\beta}^{2} \sigma_{x} .
$$

If we assume the beam has no energy spread, then under a linear focusing force, the beam's normalized emittance $\epsilon_{n}$ is a constant, so $\epsilon=\epsilon_{n} / \gamma$ is also a constant. Finally, if we use the definition of $\beta: \beta=\frac{\sigma_{x}^{2}}{\epsilon}$, we obtain

$$
\frac{1}{2} \beta \beta^{\prime \prime}-\frac{1}{4} \beta^{\prime 2}+\beta^{2} k_{\beta}^{2}=1 \text {. }
$$

[1] I. Blumenfeld, C. Clayton, F.-J. Decker, M. Hogan, C.-K. Huang, R. Ischebeck, R. Iverson, C. Joshi, T. Katsouleas, N. Kirby, W. Lu, K. Marsh, W. Mori, P. Muggli, E. Oz, R. Siemann, D. Walz, and M. Zhou, Energy doubling of 42 Gev electrons in a metre-scale plasma wakefield accelerator, Nature (London) 445, 741 (2007).

[2] M. Litos, E. Adli, W. An, C. Clarke, C. Clayton, S. Corde, D. jean pierre, R. England, A. Fisher, J. Frederico, S. Gessner, S. Green, M. Hogan, C. Joshi, W. Lu, K. Marsh, W. Mori, P. Muggli, N. Vafaei-Najafabadi, and G. Yocky,
High-efficiency acceleration of an electron beam in a plasma wakefield accelerator, Nature (London) 515, 92 (2014).

[3] W. Lu, C. Huang, M. Zhou, W. B. Mori, and T. Katsouleas, Nonlinear Theory for Relativistic Plasma Wakefields in the Blowout Regime, Phys. Rev. Lett. 96, 165002 (2006).

[4] W. K. H. Panofsky and W. A. Wenzel, Some considerations concerning the transverse deflection of charged particles in radiofrequency fields, Rev. Sci. Instrum. 27, 967 (1956).

[5] P. B. Wilson, Introduction to wakefields and wake potentials, AIP Conf. Proc. 184, 525 (1989).

[6] T. Mehrling, J. Grebenyuk, F. S. Tsung, K. Floettmann, and J. Osterhoff, Transverse emittance growth in staged laserwakefield acceleration, Phys. Rev. Accel. Beams 15, 111303 (2012).

[7] I. Dornmair, K. Floettmann, and A. R. Maier, Emittance conservation by tailored focusing profiles in a plasma accelerator, Phys. Rev. Accel. Beams 18, 041302 (2015).

[8] P. Antici, A. Bacci, C. Benedetti, E. Chiadroni, M. Ferrario, A. R. Rossi, L. Lancia, M. Migliorati, A. Mostacci, L. Palumbo, and L. Serafini, Laser-driven electron beamlines generated by coupling laser-plasma sources with conventional transport systems, J. Appl. Phys. 112, 044902 (2012).

[9] M. Migliorati, A. Bacci, C. Benedetti, E. Chiadroni, M. Ferrario, A. Mostacci, L. Palumbo, A. R. Rossi, L. Serafini, and P. Antici, Intrinsic normalized emittance growth in laser-driven electron accelerators, Phys. Rev. Accel. Beams 16, 011302 (2013).

[10] X. L. Xu, J. F. Hua, Y. P. Wu, C. J. Zhang, F. Li, Y. Wan, C.-H. Pai, W. Lu, W. An, P. Yu, M. J. Hogan, C. Joshi, and W. B. Mori, Physics of Phase Space Matching for Staging Plasma and Traditional Accelerator Components Using Longitudinally Tailored Plasma Profiles, Phys. Rev. Lett. 116, 124801 (2016).

[11] A. Aschikhin, T. J. Mehrling, A. M. de la Ossa, and J. Osterhoff, Analytical model for the uncorrelated emittance evolution of externally injected beams in plasma-based accelerators, Nucl. Instrum. Methods Phys. Res., Sect. A 909, 414 (2018), 3rd European Advanced Accelerator Concepts workshop (EAAC2017).

[12] K. Floettmann, Adiabatic matching section for plasma accelerated beams, Phys. Rev. Accel. Beams 17, 054402 (2014).

[13] R. Ariniello, C. Doss, K. Hunt-Stone, J. Cary, and M. Litos, Transverse beam dynamics in a plasma density ramp, Phys. Rev. Accel. Beams 22, 041304 (2019).

[14] C. Joshi, E. Adli, W. An, C. E. Clayton, S. Corde, S. Gessner, M. J. Hogan, M. Litos, W. Lu, K. A. Marsh, W. B. Mori, N. Vafaei-Najafabadi, B. O'shea, X. Xu, G. White, and V. Yakimenko, Plasma wakefield acceleration experiments at facet ii, Plasma Phys. Controlled Fusion 60, 034001 (2018).

[15] D. L. Bruhwiler, D. A. Dimitrov, J. R. Cary, E. Esarey, W. Leemans, and R. E. Giacone, Particle-in-cell simulations of tunneling ionization effects in plasma-based accelerators, Phys. Plasmas 10, 2022 (2003).

[16] D. J. Griffiths, Introduction to Quantum Mechanics (Pearson Prentice Hall, Upper Saddle River, NJ, 2005). 
[17] C. Huang, V. Decyk, C. Ren, M. Zhou, W. Lu, W. Mori, J. Cooley, T. Antonsen, and T. Katsouleas, Quickpic: A highly efficient particle-in-cell code for modeling wakefield acceleration in plasmas, J. Comput. Phys. 217, 658 (2006).

[18] W. An, V. K. Decyk, W. B. Mori, and T. M. Antonsen, An improved iteration loop for the three dimensional quasistatic particle-in-cell algorithm: Quickpic, J. Comput. Phys. 250, 165 (2013).
[19] S. Y. Lee, Accelerator Physics, 2nd ed. (World Scientific Publishing Co., Singapore, 2004).

[20] L. Hildebrand, W. An, X. Xu, F. Li, Y. Zhao, M. J. Hogan, V. Yakimenko, S. S. Nagaitsev, E. Adli, C. Joshi, and W. Mori, Mitigation techniques for witness beam hosing in plasmabased acceleration, 2018 IEEE Advanced Accelerator Concepts Workshop (AAC) (IEEE, New York, 2018), p. 171, https://doi.org/10.1109/AAC.2018.8659396. 